\title{
Spatiotemporal Atlas Estimation for Developmental Delay Detection in Longitudinal Datasets
}

\author{
Stanley Durrleman ${ }^{1,2}$, Xavier Pennec ${ }^{1}$, Alain Trouvé ${ }^{2}$, Guido Gerig ${ }^{3}$, \\ and Nicholas Ayache ${ }^{1}$ \\ ${ }^{1}$ INRIA - Asclepios Team-Project, Sophia Antipolis, France \\ ${ }^{2}$ Centre de Mathématiques et Leurs Applications (CMLA), ENS-Cachan, France \\ ${ }^{3}$ Scientific Computing and Imaging (SCI) Institute, University of Utah, USA
}

\begin{abstract}
We propose a new methodology to analyze the anatomical variability of a set of longitudinal data (population scanned at several ages). This method accounts not only for the usual 3D anatomical variability (geometry of structures), but also for possible changes in the dynamics of evolution of the structures. It does not require that subjects are scanned the same number of times or at the same ages. First a regression model infers a continuous evolution of shapes from a set of observations of the same subject. Second, spatiotemporal registrations deform jointly (1) the geometry of the evolving structure via 3D deformations and (2) the dynamics of evolution via time change functions. Third, we infer from a population a prototype scenario of evolution and its $4 \mathrm{D}$ variability. Our method is used to analyze the morphological evolution of $2 \mathrm{D}$ profiles of hominids skulls and to analyze brain growth from amygdala of autistics, developmental delay and control children.
\end{abstract}

\section{Methodology for Statistics on Longitudinal Data}

Many frameworks has been already proposed in medical imaging to analyze the anatomical variability of $3 \mathrm{D}$ structures like images, curves or surfaces. Less attention has been paid to the variability of longitudinal data (several subjects scanned several times). In [1, the evolution between two shapes is modeled by a geodesic deformation, which cannot be used for more than two data per subjects. In [2, shape growth is measured via the evolution of extracted features like volumes, shape or pose parameters. In [3/4, a temporal regression is proposed globally for a population, but this does not allow inter-subject comparisons. In cardiac motion analysis [5]6, spatiotemporal registration relies on 3 D-registrations between images of the same moment of the cardiac cycle and between two consecutive time-points. These works rely on time-point correspondence and do not call the labels of the time-points into question. By contrast, in longitudinal studies, subjects are scanned at ages which do not necessarily correspond. Moreover, evolutions may be delayed or advanced within a population, a key feature that we precisely aim at detecting. In [78, deformation 


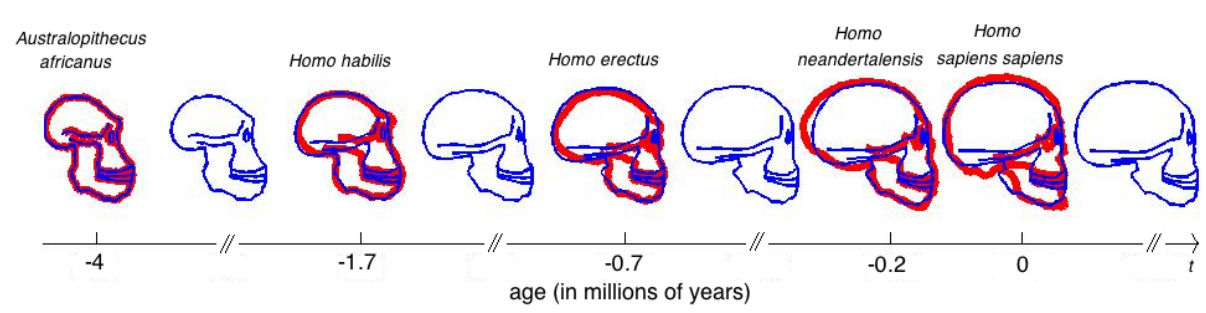

Fig. 1. Skull profile of five hominids (in red). The regression model estimates a continuous evolution (in blue) of the Australopithecus, which closely matches the data.

of cardiac motion are proposed both in space and time but they require a fine temporal sampling of the motion, whereas only few acquisitions per subjects are available in most longitudinal studies. In this paper, we propose to use a regression model to estimate a continuous evolution from data sparsely distributed in time and spatiotemporal deformations which register jointly both the 3D geometry and the scenario of evolution. Geometrical data are modeled as currents to avoid assuming point correspondence between structures. Large deformations are used which gives a rigorous framework for statistics on deformations and atlas construction 91011]. From longitudinal data, we estimate consistently the most likely scenario of evolution and its spatiotemporal variability within the population.

In this paper, we call longitudinal data a set of geometrical data (curves or surfaces, called here shapes), acquired from different subjects scanned at several time-points. We assume that the successive data of a given subject are temporal samples of a continuous evolution. We propose therefore a regression model which computes a continuous evolution which matches the data of the subject at the corresponding time points (Fig. 1). This continuous evolution allows us to compare two subjects at a given age, even if one subject has not been scanned at this age. We can also analyze how the shape varies near this age to detect possible developmental delays. We define then the spatiotemporal deformation of a continuous evolution, which consists of two deformations: (1) a morphological deformation (of the 3D space) which changes the geometry of every frame of the evolution independently of the time point and (2) a time change function (deformation of the time interval) which changes the dynamics of the evolution without changing the geometry of shapes. To avoid time-reversal, the time change function must be smooth and order preserving: it is a diffeomorphism of the time interval of interest. A $4 \mathbf{D}$ registration between two subjects looks for the most regular spatiotemporal deformation, such that the deformation of the continuous evolution inferred from the first subject maps the successive target data (Fig. 2). Eventually, we use this 4D registration framework to estimate a spatiotemporal atlas from a population, based on an $4 \mathrm{D}$ extension of the statistical model of [11. We look for a template and a continuous evolution of this template (called mean scenario of evolution), so that data of each subject 


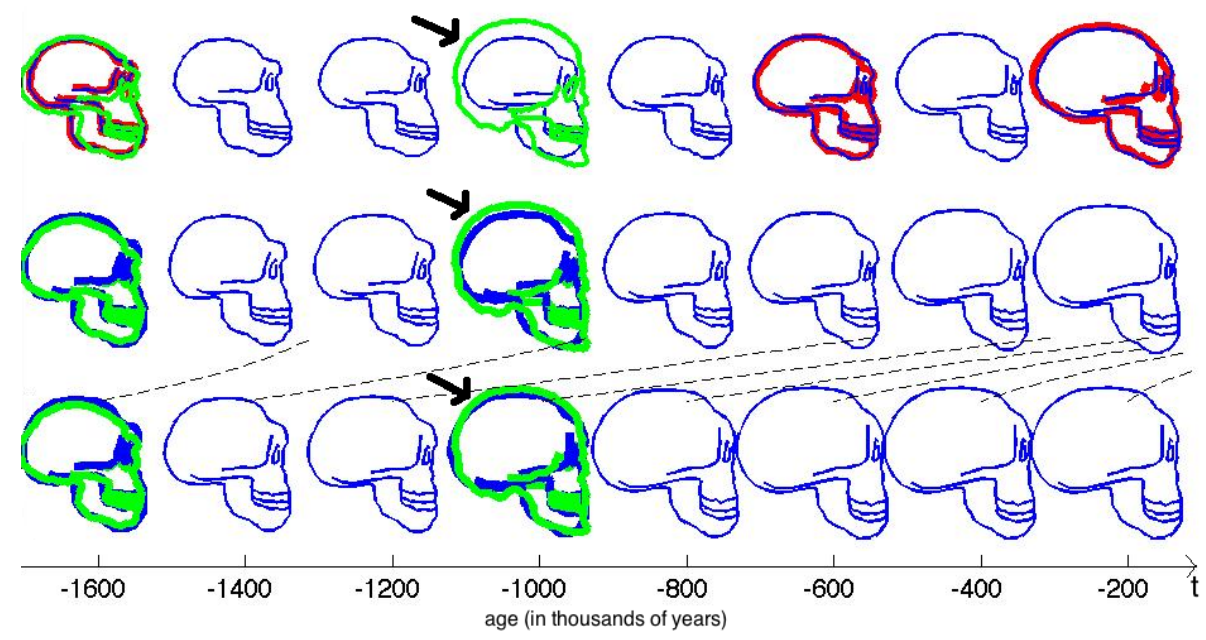

Fig. 2. Registration of the evolution \{Homo habilis-erectus-neandertalensis (in red) to the evolution \{Homo erectus-sapiens sapiens\} (in green), shifted to start at the same time. Top row: Regression of the source data (red) gives the continuous evolution in blue. Middle row: The geometrical part $\phi$ is applied to each blue frame. This shows morphological changes: the skull is larger, rounder and the jaw less prominent. Bottom row: The time change function $\psi$ is applied to the evolution of the second row. The blue shapes are moved along the time axis (as shown by dashed black lines), but they are not deformed. Black arrows show that a better alignment is achieved when one accounts both for morphological changes and a change of the evolution speed.

are temporal samples of a spatiotemporal deformation of the mean scenario. A Maximum A Posteriori estimation enables to estimate consistently the template, the mean scenario and the spatiotemporal deformations of this mean scenario to each subject.

We present the regression model in Sec. 2, 4D registration in Sec. 3 and atlas construction in Sec. 4. We focus here on the methodology and refer the reader to 12 for more computational details. In Sec. 5, we apply our method on $2 \mathrm{D}$ profiles of hominids skulls and amygdala of children scanned at 2 and 4 years.

\section{Regression Model for Shape Evolution}

We want to fit a continuous shape evolution to a set of shapes $\left(S_{i}\right)$ of the same subject acquired at different time points $\left(t_{i}\right)$. Without loss of generality, we can assume that $t_{\min }=0$ and $t_{\max }=T$. This evolving shape is equal to the baseline $M_{0}$ at time $t=0$, which may be the earliest shape of this subject or a template as in Sec. 4. The evolution has the form: $M_{t}=\chi_{t}\left(M_{0}\right)$ where $t$ varies continuously in the time interval $[0, T]$. For each $t, \chi_{t}$ is a diffeomorphism of the 3D space, such that $\chi_{0}=\mathrm{Id}$ (which leads to $\left.\chi_{0}\left(M_{0}\right)=M_{0}\right)$. The regression $\left(M_{t}\right)$ must match 
the observation $S_{i}$ at the time-points $t_{i}$, while a rigidity constraint controls the regularity of the regression. This is achieved by minimizing:

$$
J(\chi)=\sum_{t_{i}} \mathrm{~d}\left(\chi_{t_{i}}\left(M_{0}\right), S_{i}\right)^{2}+\gamma_{\chi} \operatorname{Reg}(\chi)
$$

where $\mathrm{d}$ is a similarity measure between shapes, $\operatorname{Reg}(\chi)$ a regularity term and $\gamma_{\chi}$ a trade-off between regularity and fidelity to data. Among other possible choices, we use here the large deformations of [13], and model curves or surfaces as currents 1410. Therefore, $\mathrm{d}$ is the distance between currents and $\chi$ is the solution of the partial differential equation : $\partial \chi_{t}(x) / \partial t=v_{t}^{\chi}\left(\chi_{t}(x)\right)$ with initial condition $\chi_{0}=\mathrm{Id}$. The regularity term $\operatorname{Reg}(\chi)$ is given by $\int_{0}^{T}\left\|v_{t}^{\chi}\right\|^{2} d t$. In the setting of [14, $J$ depends on time-dependent momenta $\alpha_{k}(t)$ at each samples of $M_{t}$ 's, which are used as variables for the gradient descent 1312 .

As a result, for all $t, \chi_{t}$ depends on all the constraints in the past and future. This differs from pairwise registrations between consecutive time-points. The function $\chi$ is piecewise geodesic and can be extended at all times by assuming $v_{t}^{\chi}=0$ (and hence $\chi_{t}$ constant) outside $[0, T]$. This is useful to compare this evolution with another subject which may have data outside the time interval.

\section{Spatiotemporal Pairwise Registration}

We assume now that we have successive shapes for the source subject $\left(S\left(t_{i}\right)\right)_{i}$ and for the target $\left(T\left(t_{j}\right)\right)_{j}$. As in Sec 2, we perform a regression on the source shapes which leads to a continuous evolution $S(t)_{t \in[0, T]}$. Our goal is to find a diffeomorphism of the 3D space $\phi$ and a diffeomorphism of the time-interval $\psi$ which deform the source evolution $S(t)$ into $S^{\prime}(t)=\phi(S(\psi(t)))$ such that $S^{\prime}\left(t_{j}\right)$ match $T\left(t_{j}\right)$. Thanks to the regression function, no correspondence is needed between the time points $t_{i}$ and $t_{j}$. Formally, we minimize:

$$
J(\phi, \psi)=\sum_{t_{j}} \mathrm{~d}\left(\phi\left(S\left(\psi\left(t_{j}\right)\right)\right), T_{t_{j}}\right)^{2}+\gamma_{\phi} \operatorname{Reg}(\phi)+\gamma_{\psi} \operatorname{Reg}(\psi)
$$

The spatial (resp. temporal) deformation $\phi$ (resp. $\psi$ ) is solution at parameter $u=1$ of the flow equation $\partial_{u} \phi_{u}(x)=v_{u}^{\phi}\left(\phi_{u}(x)\right)\left(\right.$ resp. $\left.\partial_{u} \psi_{u}(t)=v_{u}^{\psi}(\psi(t))\right)$. The norm of the speed vector fields $v_{u}^{\phi}$ and $v_{u}^{\psi}$ integrated for $u \in[0,1]$ defines the regularity terms $\operatorname{Reg}(\phi)$ and $\operatorname{Reg}(\psi)$ respectively. As in Sec 2 , the geometrical (resp. temporal) deformation is parametrized by momenta $\alpha$ (resp. $\beta$ ) at the points of $S\left(t_{j}\right)$ (resp. at the $t_{j}$ ), which are used as variables for the gradient descent. $\nabla_{\alpha} J(\alpha, \beta)$ is computed as for the registration of the collection of $\phi\left(S\left(\psi\left(t_{j}\right)\right)\right)$ to the $\left(T_{t_{j}}\right)$ [1410]. To compute $\nabla_{\beta} J$, we need to derive the matching term with respect to the time-points $t_{j}^{\prime}=\psi\left(t_{j}\right)$ [12. We use here a centered Euler scheme: $\nabla_{t}\|\phi(S(t))-T\|^{2} \sim\langle\phi(S(t))-T, \phi(S(t+\delta t))-\phi(S(t-\delta t))\rangle / \delta t$, where $\delta t$ is the discrete time step. We used here the fact that the shapes are embedded with a vector space (the space of currents) provided with an inner product. Note that we minimize $J$ with respect to the geometrical and the temporal parameters jointly. We do not performed alternated minimization. 


\section{Spatiotemporal Atlas Construction}

We assume now that we have a set of $N$ subjects $\left(S^{i}\right)$, provided each with temporal observations $\left(S^{i}\left(t_{j}^{i}\right)\right)_{j}$. We are looking for a template $M_{0}$ and a mean scenario of evolution of this template $M(t)=\chi_{t}\left(M_{0}\right)$, such that the observations correspond to particular moments of a spatiotemporal deformation of the mean scenario. This means that $\phi^{i}\left(M\left(\psi^{i}\left(t_{j}^{i}\right)\right)\right)$ match $S^{i}\left(t_{j}^{i}\right)$ for each subject $i$ and time $t_{j}^{i}$. Maximum A Posteriori estimation in the same setting as in [11, leads to the minimization of $J\left(\psi^{i}, \phi^{i}, \chi, M_{0}\right)=$

$$
\sum_{i=1}^{N}\left\{\sum_{t_{j}^{i}} \mathrm{~d}\left(\phi^{i}\left(\chi_{\psi^{i}\left(t_{j}^{i}\right)} M_{0}\right), S^{i}\left(t_{j}^{i}\right)\right)^{2}+\gamma_{\phi} \operatorname{Reg}\left(\phi^{i}\right)+\gamma_{\psi} \operatorname{Reg}\left(\psi^{i}\right)+\gamma_{\chi} \operatorname{Reg}(\chi)\right\}
$$

We perform a 3 step alternated minimization. If the template $M_{0}$ and the regression $\chi$ are fixed, the minimum is achieved for $N$ registrations of the mean scenario $\chi_{t}\left(M_{0}\right)$ to each subject's set of data $S^{i}\left(t_{j}^{i}\right)$, as in Sec. 3. If we fix the $N$ spatiotemporal deformations $\left(\phi^{i}, \psi^{i}\right)$ and the regression $\chi$, we need to minimize $\sum_{i, j} \mathrm{~d}\left(\Phi_{i, j}\left(M_{0}\right), S^{i}\left(t_{j}^{i}\right)\right)^{2}$, where the $\Phi_{i, j}=\phi^{i} \circ \chi_{\psi^{i}\left(t_{j}^{i}\right)}$ are 3Ddiffeomorphisms. This is exactly the estimation of an unbiased template in the setting of [11, when the deformations are given by $\Phi_{i, j}$. When the template $M_{0}$ and the $N$ spatiotemporal deformations $\left(\phi^{i}, \psi^{i}\right)$ are fixed, we need to minimize $\sum_{i, j} \mathrm{~d}\left(\phi^{i}\left(\chi_{\psi^{i}\left(t_{j}^{i}\right)} M_{0}\right), S^{i}\left(t_{j}^{i}\right)\right)^{2}+\gamma_{\chi} \operatorname{Reg}(\chi)$. This is not the regression problem stated in Sec. 2 because of the deformation $\phi^{i}$ in the matching term. To turn it into regression, we approximate the matching term $\mathrm{d}\left(\phi^{i}\left(\chi_{\psi^{i}\left(t_{j}^{i}\right)} M_{0}\right), S^{i}\left(t_{j}^{i}\right)\right)$ by $\mathrm{d}\left(\chi_{\psi^{i}\left(t_{j}^{i}\right)}\left(M_{0}\right),\left(\phi^{i}\right)^{-1}\left(S^{i}\left(t_{j}^{i}\right)\right)\right)$ (subject's shapes are matched back to the mean anatomy). This approximation is valid only for diffeomorphisms $\phi^{i}$ whose Jacobian is close to the identity. To initialize the minimization, we set $M_{0}$ as the mean current of the earliest data and set the diffeomorphisms $\chi, \phi^{i}, \psi^{i}$ to identity.

\section{$5 \quad$ Numerical Experiments}

Experiments on 2D curves relates to Sec 2 and 3 , those on surfaces to Sec. 4.

Evolution of 2D Curves. We have five 2D-profiles of hominids skulls which consist of six lines each (source: www.bordalierinstitute.com). Our regression framework infers a continuous evolution from the Australopithecus to the Homo sapiens sapiens which matches the intermediate stages of evolution in Fig. 1

Then, we register the evolution \{Homo habilis-Homo erectus-Homo neandertalensis\} to the evolution \{Homo erectus-Homo sapiens sapiens\} in Fig 2, The geometrical deformation shows that during the later evolution the jaw was less prominent and the skull larger and rounder than during the earlier evolution. The time change function shows that the later evolution occurs at a speed 1.66 times faster than the earlier evolution. This value is compatible with the growth speed of the skull during these periods (See Fig. 33): between Homo erectus and sapiens the skull volume growths at $(1500-900) / 0.7=860 \mathrm{~cm}^{3} / 10^{6}$ years, whereas 


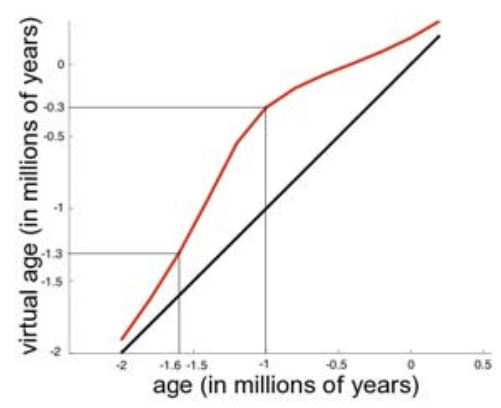

a- time change function

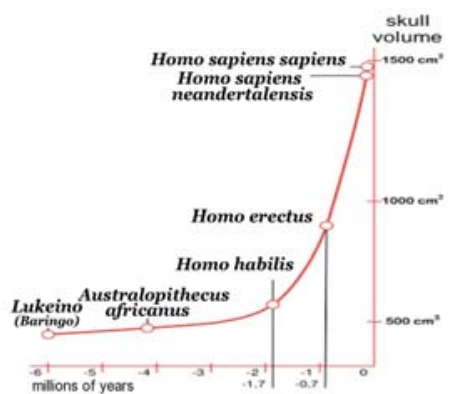

b- skull volume evolution

Fig. 3. A- time change function $\psi(t)$ of the registration in Fig. 2 (in black the reference $\psi(t)=t$ ). The slope of the curve measures an acceleration between evolutions, which is compatible with the growth of skull volume in b (source: www.bordalierinstitute.com).

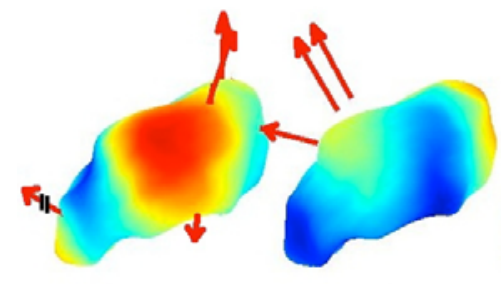

age 2

age 2.8

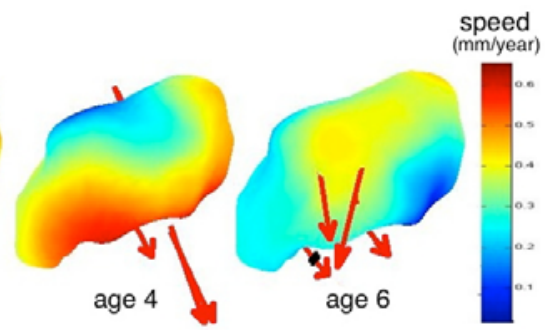

Fig. 4. Mean Scenario of the right Amygdala (right lateral part). Arrows measures the differences between age $t+0.2$ and age $t$ in the space of currents as in 16. From age 2 to 2.8 , the evolution is mainly a torque at the posterior part; then the structure becomes thicker, mostly at the superior part between age 2.8 and 4 and at the inferior between age 4 and 6 ; from age 6 the evolution is a mainly a torque at the anterior part.

between Homo habilis and neandertalensis, it growths at $(1500-600) / 1.7=$ $530 \mathrm{~cm}^{3} / 10^{6}$ years, namely 1.62 times faster.

Evolution of 3D Surfaces. We use here meshes of amygdala of the right hemisphere from 4 autistics, 4 developmental delay and 4 control children scanned twice [15. Age distribution is shown in Fig [5-a. From these data registered rigidly, we infer a template, a mean scenario of evolution of this template and the spatiotemporal evolution of this mean scenario to each subject. In the setting of [1310], the diffeomorphisms are controlled by the standard deviation of Gaussian kernel set to $15 \mathrm{~mm}$ for $\chi, \phi$ and 1 year for $\psi$; the typical scale on currents is set to $3 \mathrm{~mm}$. Trade-off $\gamma_{\chi}, \gamma_{\phi}$ were set to $10^{-3}$ and $\gamma_{\psi}$ to $10^{-6}$. An amygdala is typically $10 \mathrm{~mm}$ large. The discrete time step is set to 0.2 years.

By inspection of the companion movie of the mean scenario, one distinguishes 4 phases during growth (See also Fig. 4). Preliminary tests do not show correlations between the morphological deformations and the pathology. From the time change functions shown in Fig. 5. we cannot conclude that a subject with pathology is systematically delayed or advanced compared to controls, even at a given age. However, the curves show that the growth speed seems to follow the 

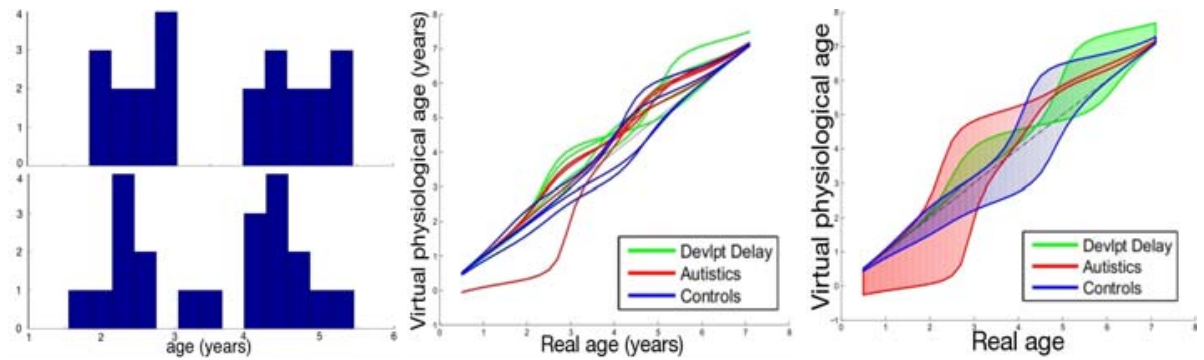

Fig. 5. Temporal deformation of the mean scenario Left: distribution of original (top) and registered (bottom) ages. Middle: time change functions for the 12 subjects. Right: First mode of variation at $\pm \sigma$ of the time change functions for each class. Autistics and controls show the same evolution pattern, but shifted in time.

same pattern, mainly an acceleration between age 2.5 and 3.5 for the autistics and between age 4 and age 5 for controls. The developmental delay also have such pattern but it occurs at a very variable age. These results suggest that the discriminative information between classes might not be inferred from the anatomical variability at a given age, but rather from variations of the growth process. These results, however, must be strengthen using larger database. The more time-points per subjects, the more constrained the mean scenario estimation. The more subjects, the more robust the statistics.

\section{Discussion and Conclusion}

In this paper, we present a generic framework to analyze variability of longitudinal data. A regression model fits a continuous evolution to successive data of one subject. 4D registrations decompose the difference between two sets of longitudinal data into a geometrical deformation and a change of the dynamics of evolution. The more acquisitions per subjects, the more constrained this decomposition. However, no constraint is imposed in terms of number and correspondence of measurement points across subjects. These pairwise registrations are used for group-wise statistics: ones estimates consistently a template, the mean evolution of this template and the spatiotemporal variability of this evolution in the population. Then, statistical measures can be derived, like the first mode of temporal deformation in Fig. 5. Further experiments have still to be performed to give more quantitative measures of variability. However, these first results suggest that pathologies might be characterized more by a particular scenario of evolution than by the anatomy at a given age. Our methodology can be used therefore to drive the search of new anatomical knowledge and to give characterization of pathologies in terms of organ growth scenario. This may be applied to the study of degenerative diseases or cardiac motion disorders.

Acknowledgments. This work was partially supported the European IP project Health-e-child (IST-2004-027749) and Microsoft Research. 


\section{References}

1. Qiu, A., Younes, L., Miller, M., Csernansky, J.: Parallel transport in diffeomorphisms distinguishes the time-dependent pattern of hippocampal surface deformation due to healthy aging and the dementia of the alzheimer's type. NeuroImage 40, 68-76 (2008)

2. Gorczowski, K., Styner, M., Jeong, J.Y., Marron, J.S., Piven, J., Hazlett, H.C., Pizer, S.M., Gerig, G.: Statistical shape analysis of multi-object complexes. Transactions on Pattern Analysis and Machine Intelligence (to appear, 2009)

3. Khan, A., Beg, M.: Representation of time-varying shapes in the large deformation diffeomorphic framework. In: Proc. of ISBI 2008, pp. 1521-1524 (2008)

4. Davis, B., Fletcher, P., Bullitt, E., Joshi, S.: Population shape regression from random design data. In: Proc. of ICCV 2007, pp. 1-7 (2007)

5. Chandrashekara, R., Rao, A., Sanchez-Ortiz, G.I., Mohiaddin, R.H., Rueckert, D.: Construction of a statistical model for cardiac motion analysis using nonrigid image registration. In: Taylor, C.J., Noble, J.A. (eds.) IPMI 2003. LNCS, vol. 2732, pp. 599-610. Springer, Heidelberg (2003)

6. Peyrat, J.M., Delingette, H., Sermesant, M., Pennec, X., Xu, C., Ayache, N.: Registration of 4D Time-Series of Cardiac Images with Multichannel Diffeomorphic Demons. In: Metaxas, D., Axel, L., Fichtinger, G., Székely, G. (eds.) MICCAI 2008, Part II. LNCS, vol. 5242, pp. 972-979. Springer, Heidelberg (2008)

7. Declerck, J., Feldman, J., Ayache, N.: Definition of a 4D continuous planispheric transformation for the tracking and the analysis of LV motion. Medical Image Analysis 4(1), 1-17 (1998)

8. Perperidis, D., Mohiaddin, R.H., Rueckert, D.: Spatio-temporal free-form registration of cardiac MRI sequences. Medical Image Analysis 9(5), 441-456 (2005)

9. Vaillant, M., Miller, M., Younes, L., Trouvé, A.: Statistics on diffeomorphisms via tangent space representations. NeuroImage 23, 161-169 (2004)

10. Durrleman, S., Pennec, X., Trouvé, A., Thompson, P., Ayache, N.: Inferring brain variability from diffeomorphic deformations of currents: an integrative approach. Medical Image Analysis 12(5), 626-637 (2008)

11. Durrleman, S., Pennec, X., Trouvé, A., Ayache, N.: Statistical models of sets of curves and surfaces based on currents. Medical Image Analysis (to appear, 2009)

12. Durrleman, S., Pennec, X., Trouvé, A., Gerig, G., Ayache, N.: Spatiotemporal atlas estimation for developmental delay detection in longitudinal datasets. Research Report 6952, INRIA (June 2009)

13. Miller, M.I., Trouvé, A., Younes, L.: On the metrics and Euler-Lagrange equations of computational anatomy. Annual Review of Biomed. Eng. 4, 375-405 (2002)

14. Vaillant, M., Glaunès, J.: Surface matching via currents. In: Christensen, G.E., Sonka, M. (eds.) IPMI 2005. LNCS, vol. 3565, pp. 381-392. Springer, Heidelberg (2005)

15. Hazlett, H., Poe, M., Gerig, G., Smith, R., Provenzale, J., Ross, A., Gilmore, J., Piven, J.: Magnetic resonance imaging and head circumference study of brain size in autism. The Archives of General Psychiatry 62, 1366-1376 (2005)

16. Durrleman, S., Pennec, X., Trouvé, A., Ayache, N.: Sparse approximation of currents for statistics on curves and surfaces. In: Metaxas, D., Axel, L., Fichtinger, G., Székely, G. (eds.) MICCAI 2008, Part II. LNCS, vol. 5242, pp. 390-398. Springer, Heidelberg (2008) 